\title{
Prediction of the amplitude of solar cycle 25 using polar faculae observations
}

\author{
Jan Janssens* \\ Solar-Terrestrial Centre of Excellence (STCE), Royal Observatory of Belgium, Avenue Circulaire 3, Brussels 1180, Belgium
}

Received 7 May 2020 / Accepted 24 December 2020

\begin{abstract}
Based on the monthly number of polar faculae, a forecast of the amplitude of solar cycle 25 (SC25) is provided, as well as a prediction of the number of solar flares. Faculae near both solar poles have been visually observed using a commercial off-the-shelf $20 \mathrm{~cm}$ Schmidt-Cassegrain telescope since 1995. The monthly averages were corrected for varying seeing conditions and the heliographic latitude of the center of the solar disk $B_{0}$. From the deduced relationship between the smoothed number of monthly polar faculae during the solar cycle minimum, and the subsequent maximum of the monthly sunspot number, a prediction has been made for the amplitude of the next solar cycle. The methodology used can be considered as a precursor technique. The expected number of M- and X-class flares was calculated based on a statistical approach. The maximum of SC25 is predicted to be $118 \pm 29$, of similar strength than the previous SC24. Also the number of M5 or stronger flares is expected to be comparable to that of the previous solar cycle.
\end{abstract}

Keywords: polar faculae / solar cycle / solar activity / solar flares

\section{Introduction}

Polar faculae (PF) usually appear as isolated bright pointlike features near the solar poles, at latitudes of about $60^{\circ}$ and higher. They have lifetimes of a few minutes to several hours, though on rare occasions lifetimes up to 5 days have been reported (Makarov \& Makarova, 1996; Saito \& Tanaka, 1957). PF are between $3 \%$ and $10 \%$ brighter than the Sun's photosphere (Makarov \& Makarova, 1996), making them dimmer than the main zone faculae which are about $9 \%-16 \%$ brighter than the photosphere (Hirayama \& Moriyama, 1979). Aside size, shape, lifetime, and location, the main difference between polar and main zone faculae is that most PF are observed around solar cycle (SC) minimum, contrary to the main zone faculae that show a peak around the time of the sunspot maximum (e.g. Sheeley, 1964). An example of polar faculae near the Sun's southern pole as observed on 12 January 2016 with the SDO/HMI instrument (Schou et al., 2012) and further processed with JHelioviewer (Müller et al., 2017) is shown in Figure 1.

Sheeley (1991) pointed out the excellent correlation existing between the number of polar faculae as recorded by the Mount Wilson Observatory (MWO), and the polar magnetic field strengths as measured by the Wilcox Solar Observatory (WSO). Hence, PF can be considered as a good proxy for

\footnotetext{
*Corresponding author: jan. janssens@oma.be
}

these polar magnetic fields, being essentially a "poor man's magnetograph" (Schatten, 2005). As these polar fields are the main ingredient in the prediction of the amplitude of the subsequent solar cycle (e.g. Schatten et al., 1978; Svalgaard et al. 2005), also the number of polar faculae could be considered as a means to predict the amplitude of the next solar cycle.

Several polar faculae data series are discussed in Petrovay (2010, 2020) and Makarov \& Makarova (1996). The longest and most thoroughly scrutinized series are the ones from the Mount Wilson Observatory as derived by Sheeley (1964, 1966, 1976, 1991, and 2008). The MWO data suffer from relatively large uncertainty margins prior to 1975 , in part because of image quality issues requiring calibration, in part because of the very limited number of observations, with one yearly data point per solar hemisphere based on only a few recordings during the most favorable viewing conditions of the respective solar poles (see Sheeley, 2008). The MWO series were further verified against SOHO/MDI observations (Scherrer et al., 1995; above $70^{\circ}$ in latitude) and calibrated to magnetic field measurements by Muñoz-Jaramillo et al. (2012, henceforth MJ12). However, correlating the maximum polar flux during SC minimum with the maximum sunspot area during the subsequent SC maximum (Muñoz-Jaramillo et al., 2013; henceforth MJ13) reveals all in all poor correlations, except when some outliers are removed (resp. correlation coefficients of 0.6 and 0.96 ). It is therefore amazing that despite the very limited availability of the polar magnetic field measurements 


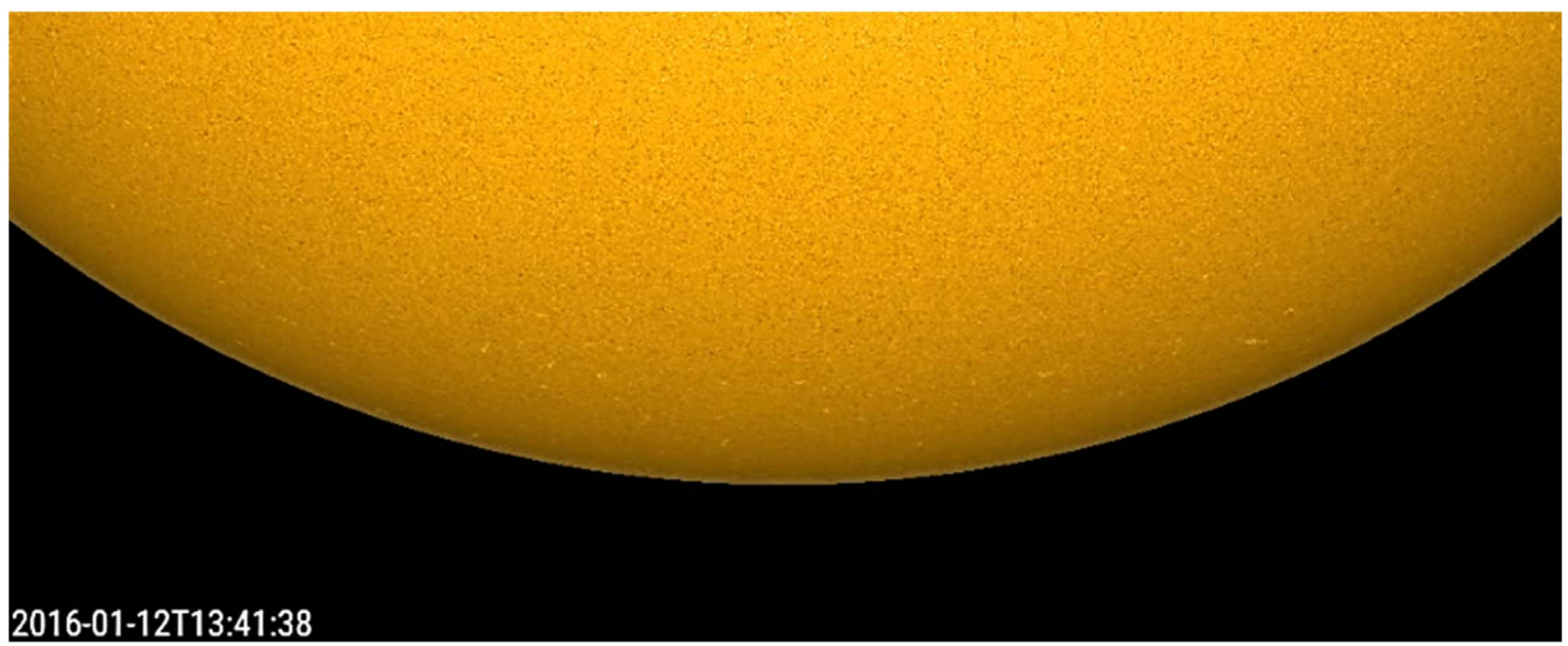

Fig. 1. Polar faculae near the Sun's southern pole as observed with the SDO/HMI instrument on 12 January 2016. Figure taken from the STCE website (see STCE Newsitem at http://www.stce.be/news/333/welcome.html). Courtesy NASA/SDO and the HMI team, and JHelioviewer.

by WSO (since 1976), solar cycle forecasts based on the polar field method (direct or proxies) proved to be consistently quite accurate for solar cycles 21-24 (Pesnell, 2016; Petrovay, 2020). This has been confirmed for other data series, such as from the National Astronomical Observatory of Japan during solar cycles 19-23 (Li et al., 2002; Tlatov, 2009).

Being an amateur solar observer since the mid 1980s, the author has been recording PF numbers using a $20 \mathrm{~cm}$ Schmidt-Cassegrain telescope since 1995. The main issue with the obtained data is the repeatability of PF observations and the data quality. This differs from most other research studies that have imagery or recordings that can be re-assessed at any point in time. The PF data presented here cannot, coming with all their shortcomings and errors. Nonetheless, the author's experience as a solar observer and the applied corrections seem to indicate that the observed polar faculae, being a proxy for the polar magnetic field strength, can be used to predict the amplitude of the next solar cycle. Hence, the next section of this paper describes how the observations were carried out and which data were gathered. In Section 3, the methodology will be discussed on how the recorded PF numbers were converted into predictions of the International Sunspot Number (ISN) for the next solar cycle. Section 4 presents and discusses the results, while Section 5 handles solar flare predictions based on the forecasted solar cycle 25 (SC25) amplitude. Section 6 summarizes the main conclusions.

\section{Observations and data}

Since September 1995, polar faculae have been observed using a commercial off-the-shelf Celestron 8 telescope. This is a $20 \mathrm{~cm}\left(8^{\prime \prime}\right)$ Schmidt-Cassegrain telescope, and it was equipped with an objective filter to dim the glare of the Sun. The solar disk was directly observed (i.e. no projection) using a $30 \mathrm{~mm}$ ocular, thus providing a magnification of $68 \times$. The obtained resolution is about 0.7 arc seconds, largely sufficient to observe PF which have typical angular sizes of 1-5 arc seconds
(Makarov \& Makarova, 1996; Saito \& Tanaka, 1957). A blue filter was screwed onto the ocular to further enhance the contrast (see e.g. Jenkins 2009). Also a shading tool, i.e. a towel or a wooden or hard-plastic note board, has been used to reduce stray light that otherwise would interfere with the observation of the polar faculae, i.e. the use of such a tool enhances the contrast between the solar limb and the dark background seen in the ocular. No records have been kept on which shading tool was used during the observation day. The complete set-up gives a contrast-rich view of the full solar disk.

The entire observation takes about 15 minutes at most (see also Saito \& Tanaka, 1957). During this time, the true north and south pole of the solar disk are found from the movement of the solar image and the known P-angle which value can be found in most astronomical almanacs. ${ }^{1}$ The area along the solar limb, about $40^{\circ}$ east and west of each pole, is then scanned for dotlike features somewhat brighter than the surrounding photosphere. The procedure is repeated once or twice to avoid double counts or missing PF. Care is also taken not to wander too far from the polar zones to avoid counting main zone faculae, in particular at the start of a new solar cycle. The observed polar faculae are counted for each pole, no pictures or drawings are taken.

During the observation, the timing and seeing conditions are noted. Solar observers readily notice that the solar image in their telescope is rarely as steady and sharp as e.g. solar images taken by spacecraft. The image always appears to be -to a certain extent- distorted, blurred, in motion, and contrast-reduced. This is due to atmospheric turbulence, which results from small temperature fluctuations in the atmosphere that give rise to turbulent mixing and in turn causes variations of the optical refractive index, deteriorating the quality of the solar image. In general, three domains in which atmospheric turbulence occurs can be distinguished (Kiepenheuer 1964):

\footnotetext{
${ }^{1}$ See e.g. the astronomical almanac published by the Royal Observatory of Belgium: https://www.astro.oma.be/en/information/ the-yearbook/
} 


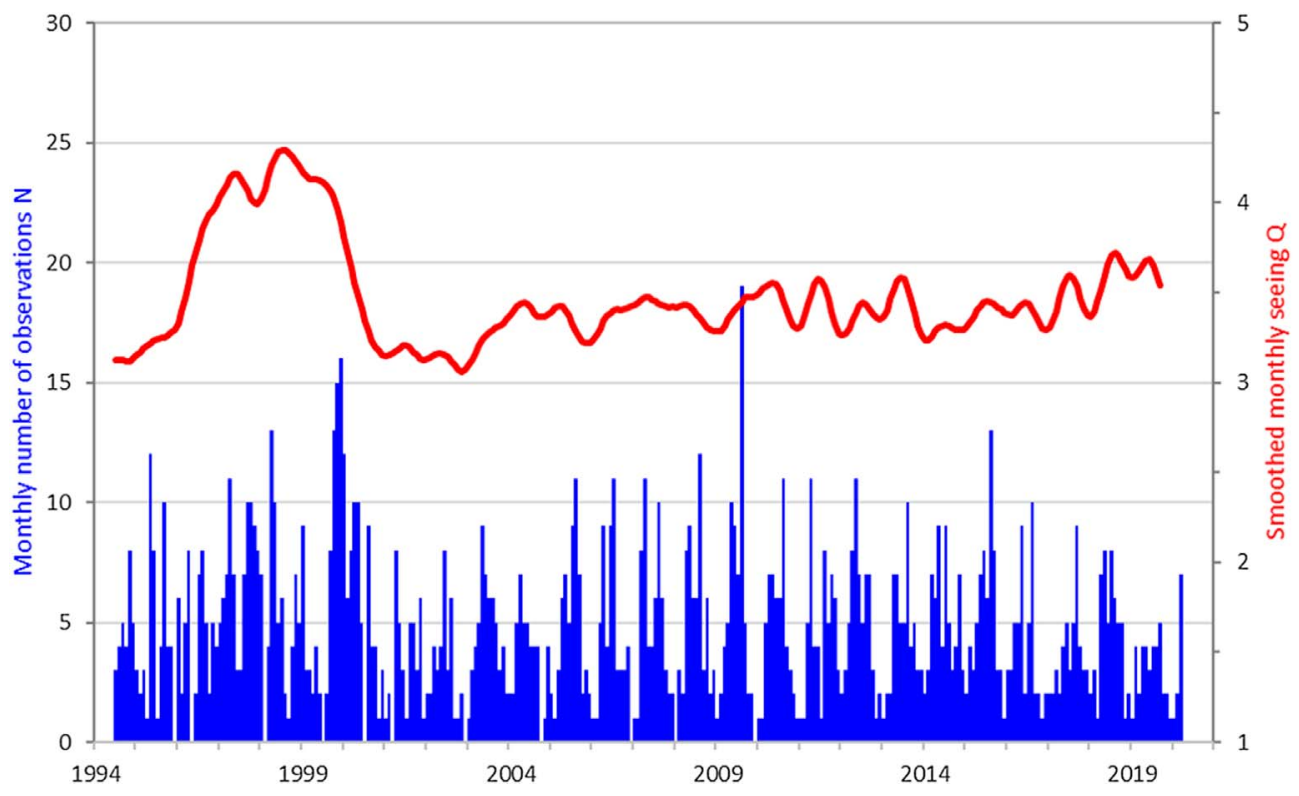

Fig. 2. Evolution of the monthly number of PF observations and monthly smoothed seeing $Q$, after having removed the days with poor seeing conditions $(Q<3)$. The period with higher $Q$-values (1996-2000) corresponds with the period observed from San Antonio, Texas, USA. A small jump in $Q$ can be seen from 2003 onwards, when it was decided to favour observations with better seeing conditions over larger number of observations.

- Local effects, such as the height of the telescope above the ground, the use of a solar dome, the type of soil, the presence of vegetation or buildings, ...;

- Mid-range effects, such as the presence (and the location relative to the observatory) of mountains, mountain ranges, nearby cities, as well as the openness of the terrain (several kilometers) surrounding the observatory, ...; and

- High-altitude effects such as the presence of cyclonic and anticyclonic systems, jet-streams, ...

All these effects have their role in the amount of atmospheric turbulence, and thus in the seeing conditions on a given moment. These "seeing" conditions were characterized using two five-step scales describing the quietness of the solar limb and the sharpness of the solar disk features by Kiepenheuer (1964). "5" means very bad conditions (blurry image and solar features hardly visible), whereas " 1 " means an excellent image (no image motion, fine details visible). The "quality scale", which combines these two parameters, was chosen by the author to assess the seeing $(Q)$. However, the scale was reversed (" 1 " being poor seeing conditions), and steps of 0.5 have been used. Indeed, because at $Q=2$ or less no suitable PF or sunspot observations can be done, and experience learned that $Q=5$ does not happen very often in Belgium, this would have left only 2 numbers (" 3 " and "4") to characterize the seeing. This was obviously not enough to properly account for the various image qualities that can be experienced.

Weather permitting, observations were mostly done during the weekend or on holidays, averaging about 5-6 per month. They were made from the author's home location near Brussels, Belgium. However, from July 1996 till June 2000, the Sun was observed - with the same instrument and set-up - from San Antonio, Texas, USA. This area has a humid subtropical climate, featuring long, hot summers and short, mild winters. This means that the location benefits from stable atmospheric conditions showing very little atmospheric turbulence for relatively long periods of time. This had its implications on the evolution of the seeing $Q$, which was much higher during that timeframe. Figure 2 shows the evolution of the monthly number of solar observations, as well as the monthly smoothed seeing $Q$. Note these concern already the corrected seeing values as obtained after removal of the days with poor seeing conditions $(Q<3$; see Sect. 3). As it became clear over the years that $\mathrm{PF}$ numbers are influenced significantly by deteriorating seeing conditions, it gradually has become a policy to avoid observing PF when $Q$ was lower than 3, thus minimizing the value for the correction factor, however thereby sacrificing in the number of PF observations.

All together there are 1617 observations of polar faculae, irregularly spread over nearly 25 years using the same instrument and set-up, and performed by the same observer. Aside the aforementioned polar faculae data series provided by professional ground-based observatories, there are also white light solar images made by satellites, giving the best quality views (unaffected by the earth's atmosphere) of the polar regions and polar faculae. However, SOHO/MDI ${ }^{2}$ (Scherrer et al., 1995) imagery is available only from May 1996 till December 2010 (Stanford's MDI webpage), and recordings by the fully operational SDO/HMI ${ }^{3}$ instrument, superseding SOHO/MDI, started only in 2010. Hence, the length of these two dataseries (on their own) is insufficient to allow for a prediction of the next solar cycle (SC25). The two datasets can be and have been combined into a series spanning a similar duration as the

\footnotetext{
${ }^{2}$ SOHO/MDI imagery at http://soi.stanford.edu/production/int_gifs. html

${ }^{3}$ SDO/HMI imagery at https://sdo.gsfc.nasa.gov/data/aiahmi/
} 
author's observations, by solving issues such as the difference in resolution between the two instruments and by analysing any line-of-sight effects. An example is e.g. Svanda et al. (2016) where the researchers investigated the contribution of the magnetic flux carried by pores to the reversal of the global magnetic field of the Sun. Nonetheless, such image processing is not the focus of the present work.

Due to the inclination of the Sun's rotation axis on the ecliptic plane, solar observers have a varying view on the solar poles throughout the year. The $B_{0}$-angle, the heliographic latitude of the center of the solar disk, reflects if observers have a better view on the northern solar pole $\left(B_{0}>0^{\circ}\right)$ or on the southern solar pole $\left(B_{0}<0^{\circ}\right)$. Over the year, $B_{0}$ varies between about $-7^{\circ}$ during February-March (best view on the southern hemisphere) and $+7^{\circ}$ during August and September (best view on the northern hemisphere). As a result, a seasonal effect exists where more PF are seen when the solar pole is directed to Earth, and less when the pole is directed away from Earth. To correct for this effect, $B_{0}=0^{\circ}$ was taken as a baseline and monthly correction factors were calculated from the changing area containing polar faculae (spherical triangles). A lower latitude boundary of $60^{\circ}\left(B_{0}=0^{\circ}\right)$ was taken for the visibility of PF (see e.g. Erofeev, 2001), so the area is bounded by the lower latitude boundary changing between about $53^{\circ}$ and $67^{\circ}$ due to the changing $B_{0}$ throughout the year. The resulting correction factors thus vary between 0.526 and 1.474. Care was taken to apply the correct factors to the corresponding hemisphere for a given month. They are multiplied with the observed PF for that month, before smoothing and corrections for seeing. The difference is obvious in the hemispheric PF numbers, where the seasonal variations are significantly reduced as will be shown in Figure 5 (Sect. 4). There's also an overall reduction in the number of PF, an obvious result of scaling to $B_{0}=0^{\circ}$.

Sunspot number data were taken from the SILSO website, ${ }^{4}$ version 2.0 (Clette et al., 2014). Polar magnetic field observations were taken from the Wilcox Solar Observatory ${ }^{5}$ (Hoeksema, 1995; Svalgaard et al., 1978). Data for the solar $\mathrm{X}$-ray flares, as measured by GOES, were taken from the NGDC $^{6}$ website up till 2017, then updated from the SWPC $^{7}$ daily event reports.

As required, the data were smoothed using Meeus formula (Meeus, 1958). This is a formula similar to the 13-month smoothing formula currently used by SILSO, but with weights for each of the months allowing smoother curves and slightly more pronounced minima and maxima.

\section{Data analysis and methodology}

The initial database spanned the period from September 1995 till March 2020, covering a total of 1617 polar faculae observations, or about $5.5 /$ month. It was quickly realized that seeing conditions influenced considerably the recorded number of PF.

\footnotetext{
${ }^{4}$ SILSO website at http://sidc.oma.be/silso/

${ }^{5} \mathrm{WSO}$ website at http://wso.stanford.edu/Polar.html

${ }^{6}$ NGDC/NOAA website at ftp://ftp.ngdc.noaa.gov/STP/space-weather/ solar-data/solar-features/solar-flares/x-rays/goes/

${ }^{7}$ SWPC/NOAA website at ftp://ftp.swpc.noaa.gov/pub/indices/events
}

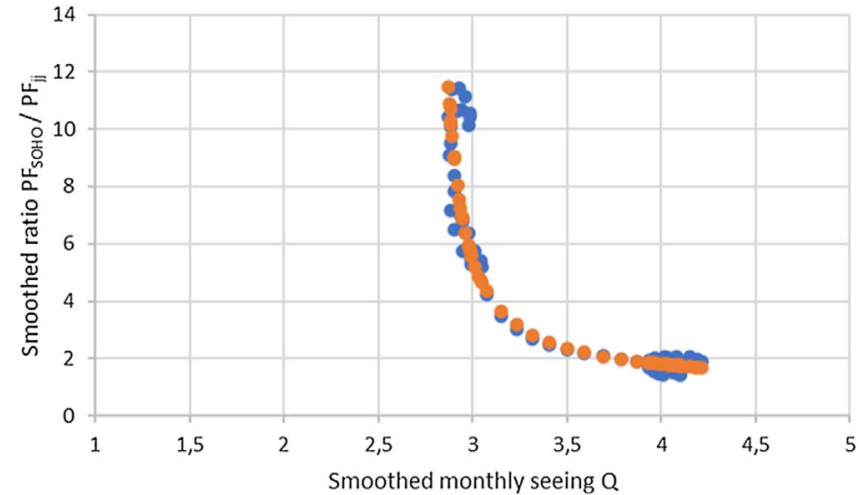

Fig. 3. Observed (blue) and approximated (orange) smoothed ratio $\mathrm{PF}_{\mathrm{SOHO}} / \mathrm{PF}_{\mathrm{JJ}}$ for the period January 1997-June 2003 as a function of smoothed seeing $Q$.

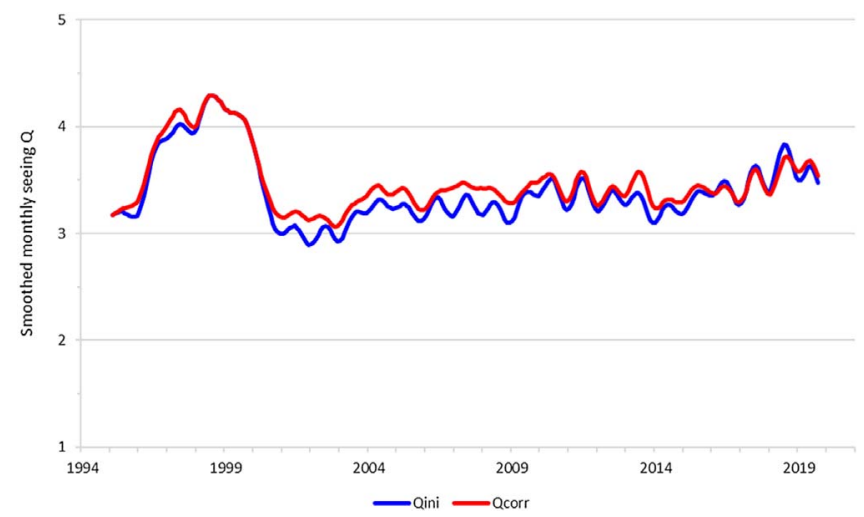

Fig. 4. Evolution of the smoothed seeing $Q$ over the period considered. The blue line $\left(Q_{\text {ini }}\right)$ concerns the data prior to the correction for days with poor seeing, i.e. before removing the observations with $Q<3$. The correction is mostly upwards (better seeing; $Q_{\text {corr }}$ ), and particularly noteworthy for the 2003-2009 timeframe.

To correct for this phenomenon, it was decided to use SOHO/MDI data as a reference, as was done by e.g. MJ12 for the calibration of the MWO PF data. Indeed, as satellites are all the time outside Earth's atmosphere, there's no atmospheric turbulence to affect the seeing conditions, thus offering a crisp solar image all the time.

The number of polar faculae per hemisphere was determined visually from SOHO/MDI imagery (limb-darkening removed) for all observing days from January 1997 till June 2003 (394), henceforth called $\mathrm{PF}_{\text {SOHO}}$. The median difference in time between $\mathrm{PF}_{\mathrm{JJ}}$ and the corresponding $\mathrm{PF}_{\mathrm{SOHO}}$ observation is $1 \mathrm{~h} 36 \mathrm{~m}$, with $75 \%$ of all observations having a SOHO image within $2 \mathrm{~h} 33 \mathrm{~m}$ of the actual observation time.

The obtained numbers for $\mathrm{PF}_{\mathrm{SOHO}}$ were then monthly averaged and Meeus smoothed and the ratio with the smoothed monthly author-based $\mathrm{PF}_{\mathrm{JJ}}$ numbers was determined.

As the aim of the observations is to have PF numbers done by the same instrument under the same seeing conditions, it was decided to have $Q=3.5$ as the reference base. Though this still means that most of the obtained monthly $\mathrm{PF}_{\mathrm{JJ}}$ numbers have to 

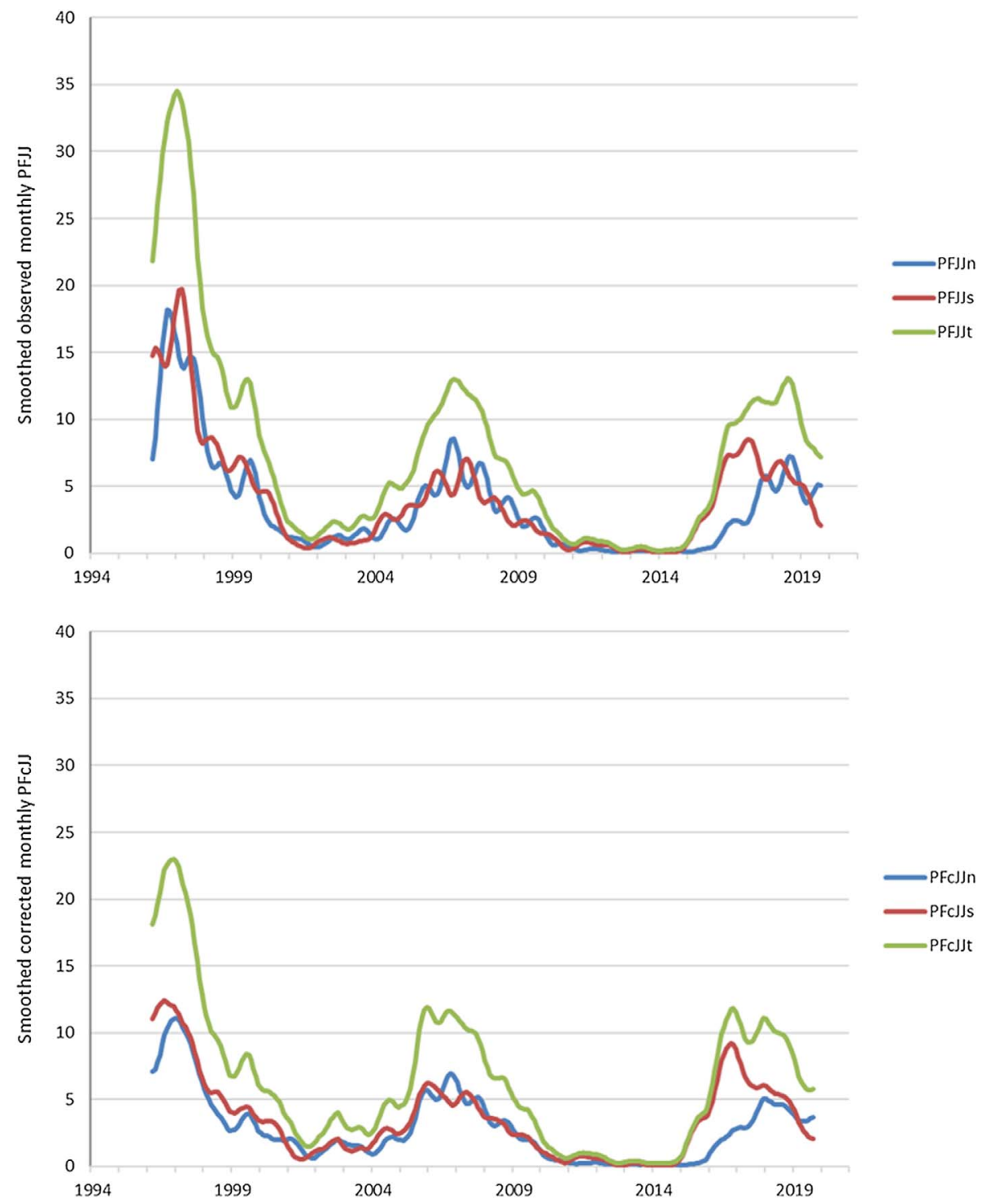

Fig. 5. Smoothed monthly values of observed $\mathrm{PF}$ (top) as recorded by the author $\left(\mathrm{PF}_{\mathrm{JJ}}\right)$ and $\mathrm{PF}$ values (bottom) which are corrected for $B_{0}$ and seeing $\left(\mathrm{PFc}_{\mathrm{JJ}}\right)$. The effect from the $B_{0}$ corrections can readily be seen as a significant reduction of the seasonal variation of the hemispheric $\mathrm{PF}$ numbers. The seeing correction concerns both the removal of days with poor seeing and a baselining of all observations to $Q=3.5$. Both figures show the hemispheric values (North " $n$ " in blue, South "s" in red) and the totals (green).

be corrected for the varying seeing, it also allows the correction factors to remain relatively small. For this, first a scatter plot with smoothed seeing $Q$ versus the ratio $\mathrm{PF}_{\mathrm{SOHO}} / \mathrm{PF}_{\mathrm{JJ}}$ was created. Smoothed monthly data of $Q$ and the ratio $\mathrm{PF}_{\mathrm{SOHO}} / \mathrm{PF}_{\mathrm{JJ}}$ for the period January 1997 till June 2003 were taken. These are represented by the blue values in Figure 3. A best-fitting curve was established, with the ratio equal to $(Q-1.80) /$ $(Q-2.78)$. These are the orange squares in Figure 3. For $Q=3.5$, the reference value of this ratio then equals 2.3611 .

The final corrected PF numbers as observed by the author are then $\mathrm{PFc}_{\mathrm{JJ}}=\mathrm{PF}_{\mathrm{JJ}} \times(Q-1.80) /(2.3611 \times(Q-2.78))$. If for a certain month the smoothed $Q=5$, then $\mathrm{PF}_{\mathrm{JJ}}$ would be corrected by a factor of 0.611 . If smoothed $Q=3$, then $\mathrm{PF}_{\mathrm{JJ}}$ would be increased by a factor 2.310 . If the smoothed $Q$ would be exactly 3.5 , then the correction factor would be 1 and $\mathrm{PFc}_{\mathrm{JJ}}=\mathrm{PF}_{\mathrm{JJ}}$.
The correction factor can sometimes take on large values, e.g. the correction factor can become larger than 10 which happens for values of $Q<2.88$. This can be seen in the reduction curve (orange) of Figure 3 which is quite steep towards lower $Q$-values, reflecting an observational fact which one can readily notice during an observing session with varying seeing: During periods of bad seeing, the PF which were visible during the brief moments of better seeing are completely washed out, raising some concerns over the true number of $\mathrm{PF}$ that were actually visible during an observing session with continuously bad seeing. Indeed, from the comparative analysis with the SOHO/MDI data outlined above, the percentage with $0 \mathrm{PF}$ for seeing conditions $Q=2,2.5$, and 3 is resp. $100 \%, 57 \%$ and $29 \%$. For all other $Q$-values, that percentage was at or below 5\%. For the $\mathrm{SOHO}$ data, there were no days with 0 PF. Therefore, all observations with $Q<3$ were removed. 
After this correction, 1383 observations remained (4.69/month) and the average seeing over the entire period had increased from 3.34 to 3.48 . Figure 4 shows the smoothed seeing for the original observations (blue line) and the smoothed corrected values (red line), i.e. after removal of the observations with $Q<3$. The evolution is mostly upwards (better seeing conditions), with exception of the years 2017-2018 when there were long periods of very good seeing (long summers with stable atmospheric conditions). The most noteworthy change can be seen during the 2004-2009 period, i.e. during the SC23SC24 transit. The smoothing formulas were adapted accordingly to accommodate occasional months with no PF observations (11 months, all prior to 2010).

Though there exist concrete guidelines for the quantification of the observing conditions (Kiepenheuer, 1964), the quantification of these conditions during an observation ( $Q$ factor) may seem somewhat subjective and depend on the experience of the observer. An alternative method was tried out, in the sense that a polynomial was used to quantify the relation between $\mathrm{PF}_{\mathrm{JJ}}$ and $\mathrm{PF}_{\mathrm{SOHO}}$. A 5th degree polynomial gave the best results $\left(r^{2}=0.966\right)$. Applying this formula to the observed total polar faculae count (already corrected for $B_{0}$ ) resulted in the total $\mathrm{PF}_{\mathrm{JJ}}$ being about 2.2 to 2.5 times higher than the initial values. This is expected as the corrections are basically bringing the observed values to the best possible seeing conditions, i.e. $Q=5$. From the observed maxima in PFt, the maximum for SC25 as derived from the polynomial is only $3 \%$ higher than the seeing corrected value (Sect. 4). In view of this small difference, and because the seeing influence is more prominently visible when using the ratio methodology, it was elected to stick to the seeing-corrected result.

\section{Results and discussion}

Figure 5 shows the smoothed observed $\mathrm{PF}_{\mathrm{JJ}}$ and corrected $\mathrm{PFc}_{\mathrm{JJ}}$. The $\mathrm{PFc}_{\mathrm{JJ}}$ values have been corrected for the varying $B_{0}$ and seeing, the latter by removing all the observations with $Q<3$, as well as by applying the formula to bring all monthly PF numbers to the same level of seeing $(Q=3.5)$. The effect from the $B_{0}$ corrections can readily be seen as a significant reduction of the seasonal variation of the hemispheric PF numbers. There's an overall reduction in the PF number over the entire period by $12-18 \%$, a result from $B_{0}=0^{\circ}$ chosen as the reference. The seeing influence is different for the three cycles. For SC23, the influence from the seeing correction (Fig. 3) decreases the maximum $\mathrm{PF}$ number further by another $23 \%$, from 30.2 to 23.0. For the PF numbers preceding SC24 and SC25 however, there's a slight increase of respectively $+11 \%$ (from 10.7 to 11.9 ) and $+4 \%$ (from 11.4 to 11.8 ). The contributions of the two corrections on the total PF number can be seen in Figure 6. The latest observations indicate that the PF numbers have considerably declined after 2018, and this at both solar poles.

As described in e.g. Petrovay (2020), a linear relationship is assumed between the observed number of PF and the subsequent maximum of the Meeus smoothed monthly sunspot number (SILSO, version 2.0). Based on the maximum $\mathrm{PFc}_{\mathrm{JJ}}$ recorded during the 2016-2019 period, a maximum sunspot number of 117.9 (rounded to 118) is obtained for SC25 (Fig. 7). This indicates that the intensity of the upcoming solar cycle would be very similar to SC24, excluding a cycle strength similar to SC23 and making a Dalton-like minimum such as during the early 19th century very unlikely.

Because there are only 2 data points (for SC23 and SC24), the correlation coefficient is 1 implying an uncertainty margin of 0 . This prevents of course any significant conclusion about the prediction for SC25. To have an idea of the uncertainty margin, a prediction for SC24 was made by establishing a simple linear relationship using the polar magnetic fields from SC22 and SC23 (2 data points) as observed and smoothed by the Wilcox Solar Observatory. The obtained sunspot number maximum for $\mathrm{SC} 24$ was 13 above what was observed (131.4 predicted for 118.2 observed). Another method is to compare the MWO maximum PF data with the maximum ISN of the subsequent solar cycle, as this is the longest existing PF database (since 1906). Taking the original data (Sheeley, 2008) and the calibrated data (MJ12), and excluding two outliers (SC16 and 20) in both series, respective standard deviations of 25.4 and 28.9 are obtained ( $p$-values both significant at 0.01). As a result, for the predicted SC25 amplitude based on $\mathrm{PFc}_{\mathrm{JJ}}$, an error margin of 29 has been adopted.

MJ12 obtained a very good correlation between the automatically derived MDI PF (above $70^{\circ}$ latitude, 19962010), the MWO PF (after several calibrations for the different observing campaigns), and the WSO polar MF strength. Therefore, as a consistency check, a direct comparison between the hemispheric yearly $\mathrm{PFc}_{\mathrm{JJ}}$ and the WSO MF data has been made (1996-2019). Figure 8 shows the yearly WSO polar MF and the signed $\mathrm{PFc}_{\mathrm{JJ}}$. The sign used for the $\mathrm{PFc}_{\mathrm{JJ}}$ has been taken the same as that from WSO for the respective year. There's a good correlation between the maximum number of $\mathrm{PF}$ and the maximum WSO polar MF strength during the maximum years. At the same time, there also seems to be a deficit in the number of $\mathrm{PF}$ as compared to the polar MF during the transit years from minimum to maximum MF strength and back from maximum to minimum. This seems to suggest that $\mathrm{PFc}_{\mathrm{JJ}}$ are lacking the fainter variety of the PF, which are detected in the MWO and MDI imagery.

As an analogy to MJ12 (their Fig. 11b), where the calibrated MWO PF is shown against WSO polar MF $(G)$ for the years 1976-2010, the signed yearly hemispheric $\mathrm{PFc}_{\mathrm{JJ}}$ has been compared to the corresponding yearly WSO polar MF strength for the years 1996-2019. For all data, so including those from the transit years, the linear coefficient is 0.099 and the correlation coefficient 0.90 (In MJ12 respectively 0.08 and 0.90). Removing the transit years for $\mathrm{PFc}_{\mathrm{JJ}}$, which was done essentially by removing all yearly hemispheric $\mathrm{PFc}_{\mathrm{JJ}}<3$, results in a linear coefficient of 0.092 and an $r$-value of 0.96 . Removing all yearly hemispheric $\mathrm{PFc}_{\mathrm{JJ}}<5$, improves the correspondence with the MJ12 results even further: a linear coefficient of 0.085 and an $r$-value of 0.99 . Though the number of outliers is further reduced this way, the better results come at the expense of reducing the range of applicability (respectively only half and a quarter of the original data remain). The general conclusion is then that the yearly $\mathrm{PFc}_{\mathrm{JJ}}$ are a good estimate for polar MF strength but only for $\mathrm{PFc}_{\mathrm{JJ}}>5$. As a result, for the prediction of the amplitude of the next solar cycle, the $\mathrm{PFc}_{\mathrm{JJ}}$ data remain a valid alternative to the WSO polar MF strength precisely because the maximum $\mathrm{PFc}_{\mathrm{JJ}}$ are taken.

Figure 5 also shows the evolution of the hemispheric number of PF. Of note is the fast and strong rise of the number of southern PF, and the much slower rise of the number of 


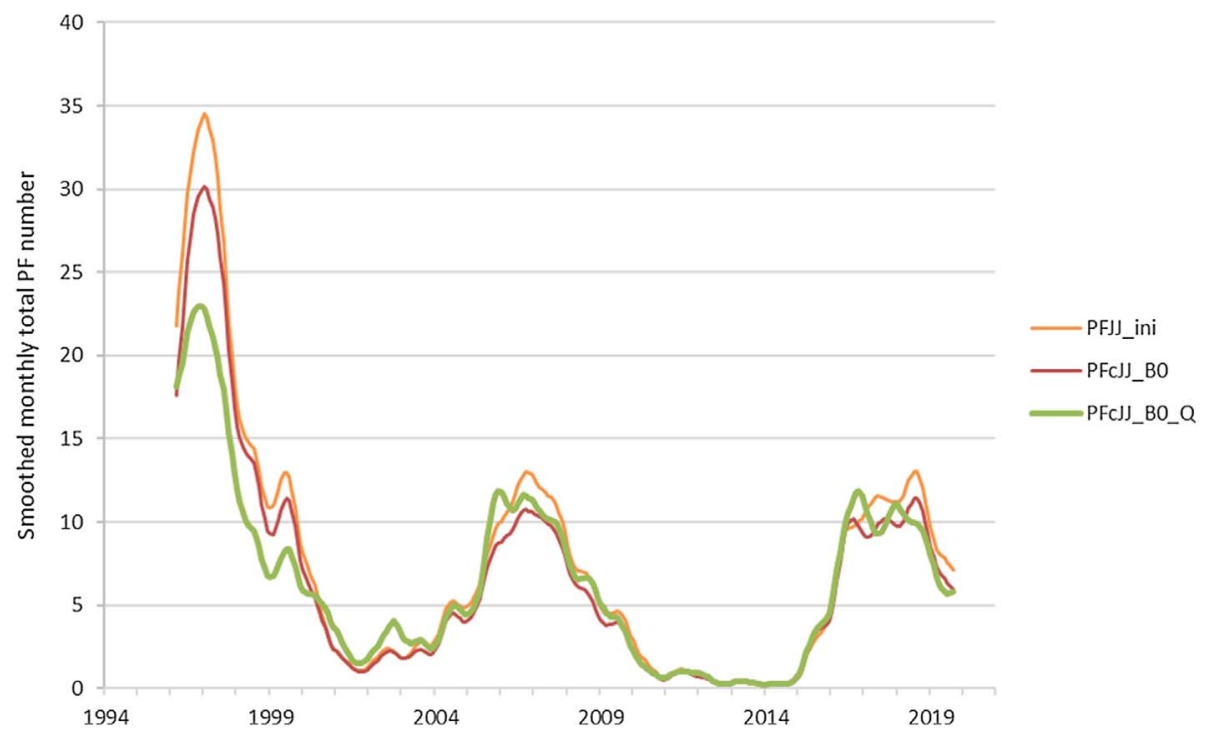

Fig. 6. The smoothed initial monthly number of $\mathrm{PF}$ after removal of the observations with $Q<3$ (orange curve), the overall reduced number of $\mathrm{PF}$ following the correction for $B_{0}$ (brown curve), and the final corrected $\mathrm{PFc}_{\mathrm{JJ}}$ (green curve) after an additional correction for the varying seeing (see Fig. 3). The correction for $B_{0}$ results in an overall decrease of the PF numbers by $12-18 \%$. The correction for seeing decreases the PF numbers prior to SC23, but mostly increases the PF numbers prior to SC24 and SC25.

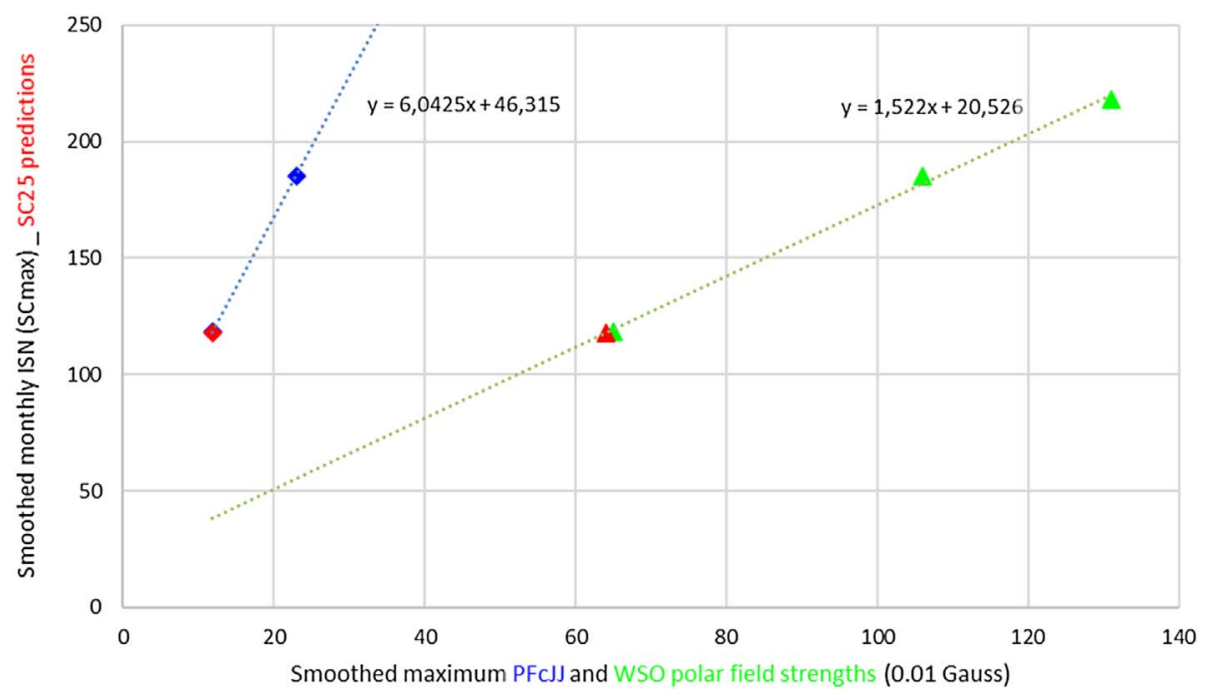

Fig. 7. Meeus smoothed maximum $\mathrm{PFc}_{\mathrm{JJ}}$ (blue diamonds) and WSO smoothed maximum polar field strengths (0.01 Gauss; green triangles) versus the maximum smoothed monthly sunspot number (ISN) for the respective solar cycles. The square of the correlation coefficient $\left(r^{2}\right)$ is unity for the $\mathrm{PFc}_{\mathrm{JJ}}$ (only 2 datapoints), and 0.9969 for WSO (3 datapoints; St.Dev. 3) with a p-value of 0.036, significant at the 5\% level. The $\mathrm{SC} 25$ predictions based on the currently observed maximum $\mathrm{PFc}_{\mathrm{JJ}}$ and WSO polar field strength are in red. Both methods give nearly identical amplitudes for the upcoming SC, predicting maximum smoothed monthly sunspot number for SC25 of 117.9 (rounded to 118), which is almost the same as the observed SC24 maximum (118.2). Hence, both prediction methods seem to indicate that SC25's amplitude will be similar to that of SC24.

northern PF. This may be due to the unusual polar field reversal during SC24 (Janardhan et al., 2018), which happened very quickly at the southern solar pole, but took a long time at the northern pole. However, it is premature to transpose this evolution of the hemispheric PF into a conclusion that SC25 might be e.g. a double-peaked cycle. As mentioned in
Charbonneau (2020), there are many solar dynamo features, such as magnetic diffusivity, circulation speed, cycle memory, ... that may affect the strength and timing of the hemispheric sunspot numbers. Therefore, no further efforts have been made in this paper to expand on the forecast of the hemispheric evolution of SC25. 


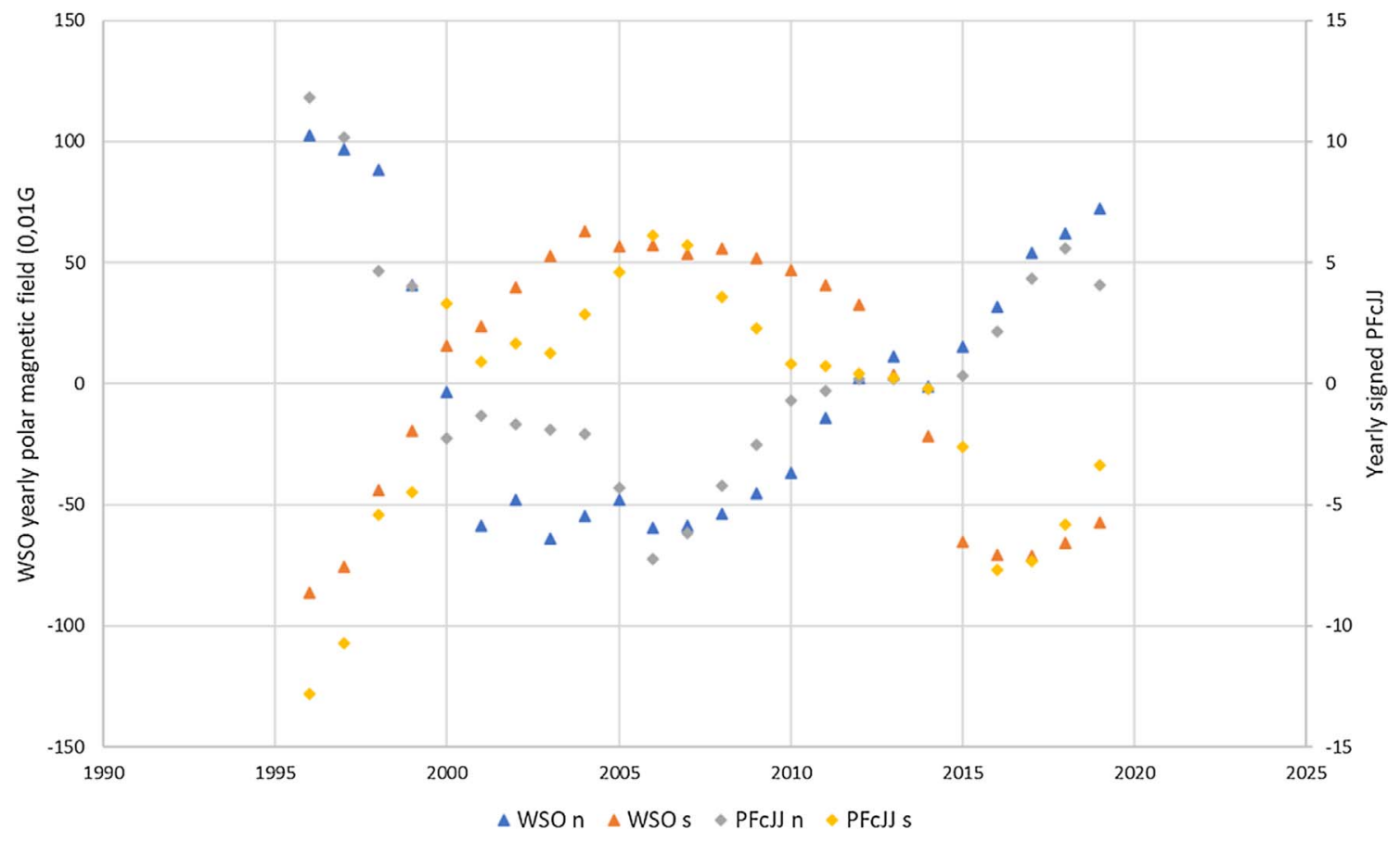

Fig. 8. Comparison between the signed hemispheric yearly $\mathrm{PFc}_{J \mathrm{~J}}$ and the WSO MF data (1996-2019). The sign used for the PFc $\mathrm{c}_{\mathrm{JJ}}$ has been taken the same as that from WSO for the respective year. There's a good correspondence between the maximum number of $\mathrm{PF}_{\mathrm{JJ}}$ and the maximum WSO polar MF strength during the maximum years. A deficit in the number of $\mathrm{PFc}_{\mathrm{JJ}}$ as compared to the WSO polar MF can be seen during the transit years between the periods of minimum and maximum MF strength. This seems to suggest that $\mathrm{PFc}_{\mathrm{JJ}}$ are lacking the fainter variety of the PF, which are detected in the MWO and MDI imagery.

\section{Flaring activity}

In their analysis of superactive regions (SAR), Chen et al. (2011) pointed out that during solar cycles 21-23 45 SARs were responsible for $44 \%$ of all X-class solar flares. Otherwise said, as about 10.000 sunspot regions over this 30-year period emerged, just half of a percent of them produced nearly half of all the strong solar flaring events. For SC24, with only 5 active regions that produced 3 or more X-class flares during their transit of the solar disk, the statistics are very similar (resp. $0.3 \%$ and $41 \%$ ). These numbers testify of the erratic nature in the timing and the number of these strong solar flares, and pose a challenge on any long-range prediction of these eruptive events.

In order to get an idea of the flaring activity during SC25, a statistical approach was taken and tailored to the ISN amplitude of 118 expected for SC25. The study is limited to M- and $\mathrm{X}$-class flares, as these are the source of the radio black-outs described by NOAA's R-scale (Poppe, 2000). In the current study, the R-classes have been binned in three groups such that each has a sufficient number of flares: M1-M4 (R1), M5-M9 (R2), and the X-class flares (R3-R5).

First of all, it is noteworthy that there seems to exist a reasonable correlation between the amplitude of a solar cycle (ISN) and the total number of $\mathrm{M}$ - and X-class flares during the respective solar cycle (Fig. 9). The correlation coefficient $r$ does not drop below 0.99 , with $p$-values significant at the $1 \%$ level. Applying the derived regression formulas on the expected SC25 amplitude, a total of $745 \pm 58 \mathrm{M}$ - and X-class flares can be expected: $611 \pm 68$ M1_4 flares, $82 \pm 7$ M5_9 flares and $52 \pm 5 \mathrm{X}$-class events.
With GOES-16 coming into operation, NOAA/SWPC has stopped applying a correction factor to the recorded X-ray fluxes ("0.7" for the XRSB Long Channel covering from 0.1 to $0.8 \mathrm{~nm}$ ). Therefore, the X-ray fluxes reported by previous GOES satellites are lower than the currently reported ones. ${ }^{8}$ This basically means that a C7 flare in the old system counts as a M1 flare in the new reporting system (GOES16 and beyond). The strongest flare in the database, an X28 event observed on 4 November 2003, would now count as an X40 flare. An analysis of the entire existing database at NOAA/ SWPC reveals that, averaged over the last 4 solar cycles, there would be $55 \%$ more M-class flares and 52\% more X-class flares than with the re-scaling factor of 0.7 applied.

Correcting for this rescaling factor, the relationship between the amplitude of a solar cycle (ISN) and the total number of Mand X-class flares during the respective solar cycle remains solid (Fig. 9 bottom). The correlation coefficient $r$ slightly improves for the M1_4 and X-class bins with the $p$-value well below 0.01. For the M5_9 bin, the $r$ - and $p$-values slightly weaken, from 0.9923 and 0.008 to respectively 0.9777 and 0.022 . Applying the derived regression formulas on the expected SC25 amplitude, a total of $1151 \mathrm{M}$ - and X-class flares can be expected: $957 \pm 72$ M1_4 flares, $118 \pm 17$ M5_9 flares and $76 \pm 1$ X-class events.

Contrary to the number of flares, it is far more difficult to get an idea of the timing of these flares, due to the erratic nature

\footnotetext{
${ }^{8}$ See the NOAA/SWPC notes at https://www.swpc.noaa.gov/ news/goes-14-15-are-now-storage-mode and https:/www.swpc. noaa.gov/sites/default/files/images/GOES\%2016\%20XRS\%20Updates $\% 20$ and $\% 20$ Status.pdf
} 

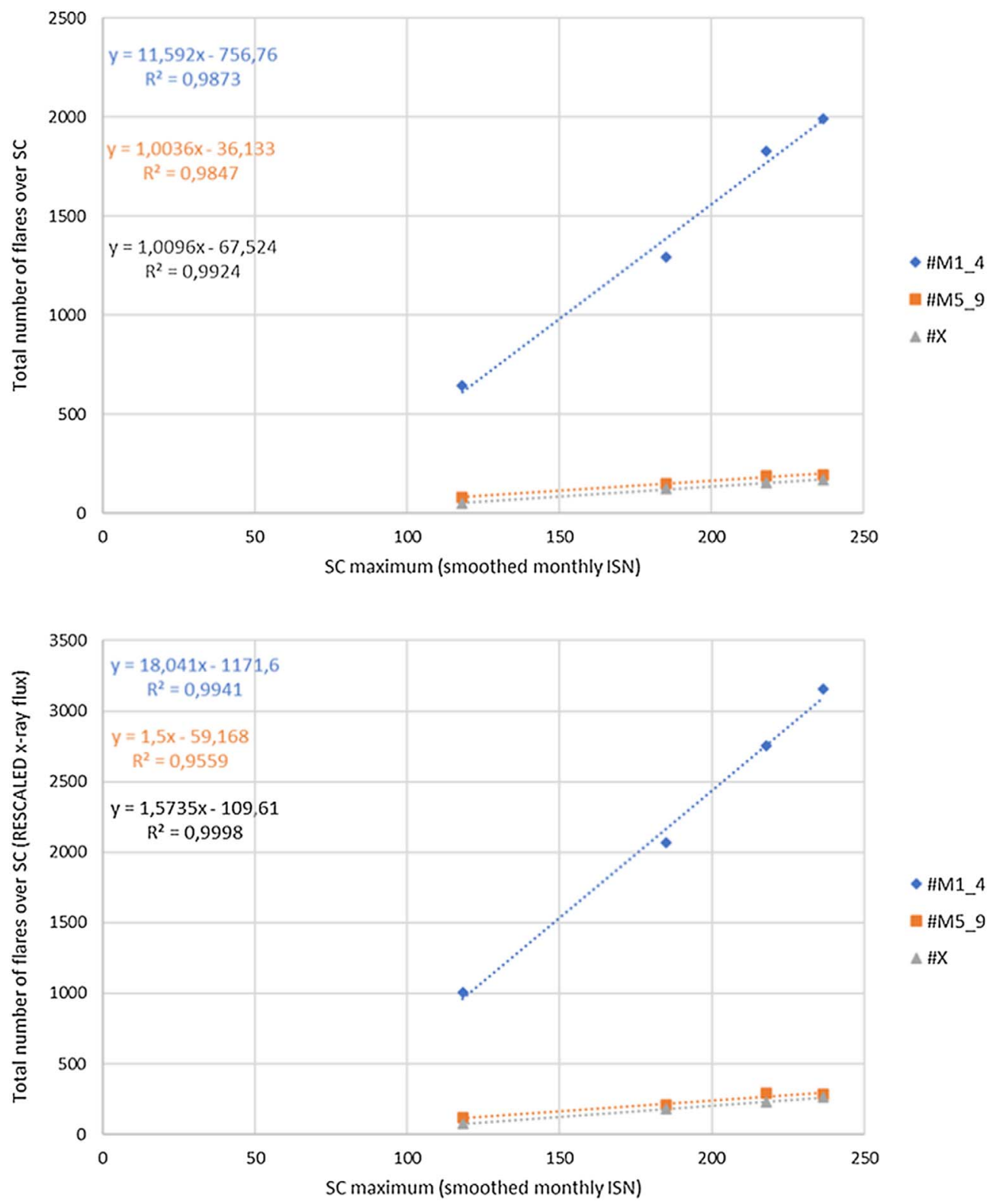

Fig. 9. (Top) The total number of flares per solar cycle in each category, i.e. M1 to M4 (blue), M5 to M9 (orange), and X (grey), against the maximum smoothed monthly sunspot number of the respective cycle. The equations of the trendline and the square of the correlation coefficient $\left(r^{2}\right)$ for each flare category are in the upper left corner. (Bottom) The same as in the top figure, but this time without the rescaling factor of 0.7 applied over the solar cycles. There's an increase of about 55\% in the number of M-class flares, and 52\% in the number of X-class flares. The correlations and $p$-values improve a bit for the M1_4 and X-class bin, but slightly decrease for the M5_9 bin ( $r$ and $p$ from 0.9923 and 0.008 to 0.9777 and 0.022).

of the solar flare production, as already outlined at the beginning of this section. Balogh et al. (2014) in their discussion on solar activity indicators (their Sect. 3 and Fig. 3) already noted that while the occurrence rate of solar flares is linked to solar activity as measured by e.g. the monthly sunspot number, there are significant differences in terms of the phase of the sunspot cycle, as well as its intensity and frequency distribution. Indeed, while the monthly rates of C-class solar flares (relatively small flares, with mostly little terrestrial effects) varies with an approximate periodicity of 11 years, the relatively small number of the largest flares (X-class) have an occurrence frequency that only indicates that such strong flares usually do not occur during solar cycle minimum activity, but further do not match the profile of other activity indicators such as the sunspot numbers.

To partially alleviate this problem, one can take the yearly instead of the monthly numbers. Figure 10 shows the observed yearly ISN, and the observed yearly number of M- and X-class flares for the period 1976-2019 (with the rescaling factor removed for the X-ray flux). For SC21-24, there's now a better correspondence between the flare numbers and the solar cycle, though there's still some out-of-phase for SC21 and somewhat excessive numbers during the declining portion of SC23. Of 


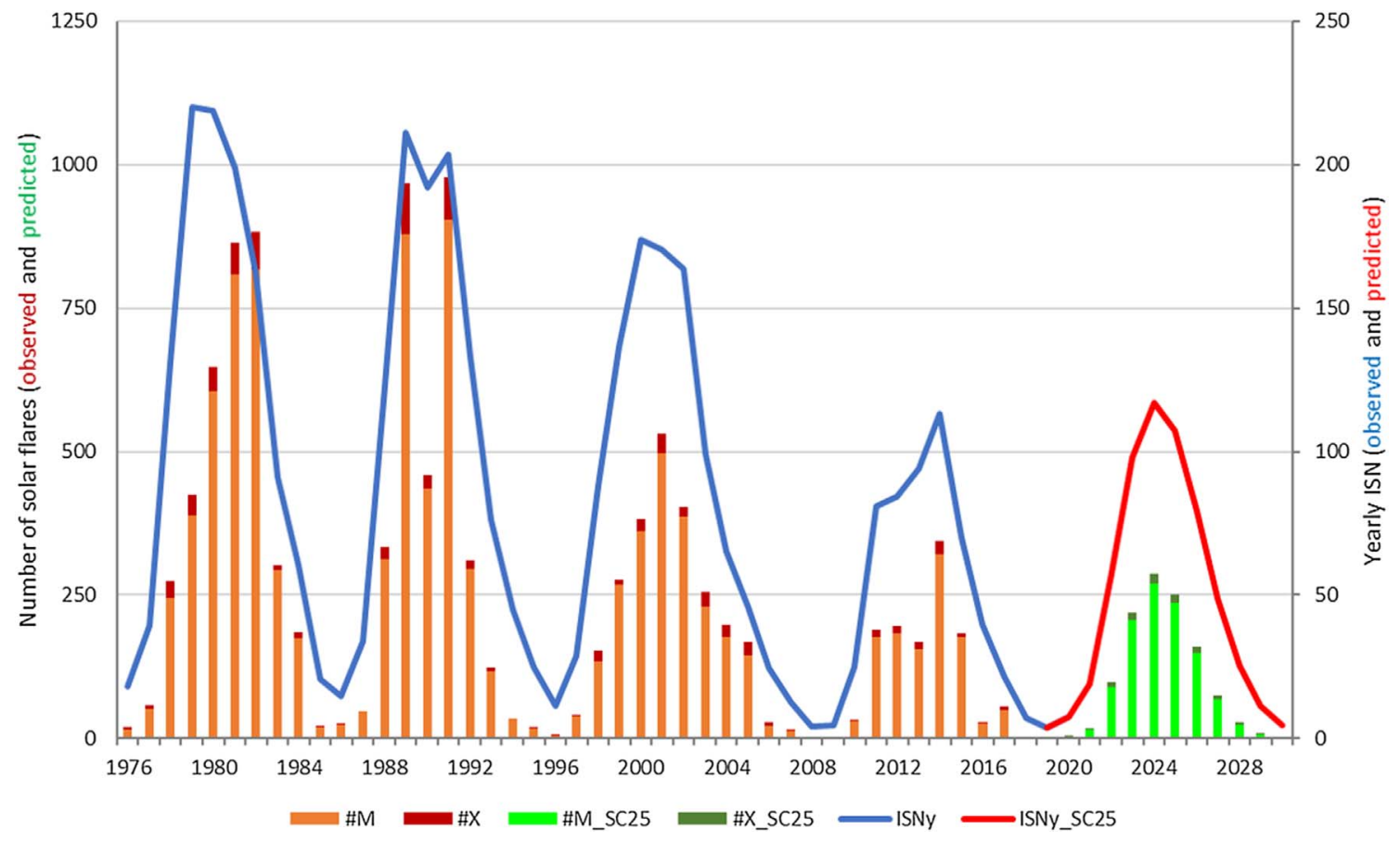

Fig. 10. This graph shows the observed (SC21-24) and predicted (SC25) yearly ISN (ISNy), as well as the yearly number of observed and predicted M- and X-class flares. The SC25 flare numbers are expected to be similar to SC24, and 3-4 times smaller than SC21 and 22. For the expected maximum year for SC25 (2024), $270( \pm 70)$ M-class flares and $17( \pm 8)$ X-class flares can be expected. The flare numbers as well as their distribution over SC25 can be quite different from the ones here displayed, if e.g. the final sunspot numbers deviate significantly from the sunspot curve used here, and may be further skewed with the occurrence of SARs. Nonetheless, the total numbers are not expected to deviate significantly for the ones expected over the entire SC25, i.e. $1075 \mathrm{M}$ - and 76 X-class flares.

note too is that the Gnevyshev gap is significantly more pronounced in the flare numbers (about $-50 \%$ decrease from the maximum peak) than that in the smoothed sunspot number evolution (about $-15 \%$ ), as can be seen for SC22. This effect is also visible in the monthly numbers. Physical models discussing the source of the Gnevyshev peaks and gaps can be found in e.g. Norton \& Gallagher (2010), Georgieva (2011) and Karak et al. (2018).

For SC25 (2020-2030, Fig. 10), the ISN curve is a generic sunspot curve to guide the eye, as derived using the parametrized equations from Hathaway et al. (1994), with a maximum monthly ISN of 118 (the value predicted from the $\mathrm{PFc}_{\mathrm{JJ}}$ ) at month 56 and then yearly averaged. The predicted number of M- and X-class flares are derived from a power law based on the yearly ISN and the recorded yearly flare numbers from 1976 to 2019. These values are then rescaled so that their sum over the 11 years matches the overall flare numbers expected for the entire next SC25 (i.e. $1075 \mathrm{M}$ - and $76 \mathrm{X}$-class flares). As a result, for the maximum year 2024, $270( \pm 70)$ M-class flares and $17( \pm 8)$ X-class flares can be expected. It should be very clear that these numbers are not real forecasts, but are just intended to give the space weather end user an idea of what to expect in terms of strong flares and how they might be distributed over the solar cycle. As the here presented numbers are indeed a function of the ISN, and if SC25 deviates from the typical solar cycle curve as was the case for e.g. SC24, then of course the final recorded flare numbers can be significantly differ from the ones displayed in Figure 10. SARs can skew these numbers even further. Nonetheless, it is also clear that the flare numbers for SC25 are thought to be a factor 3-4 smaller than those produced by SC21 and 22, and expected to be similar to those from $\mathrm{SC} 24$.

\section{Conclusions}

Polar faculae have been observed with a $20 \mathrm{~cm}$ SchmidtCassegrain telescope since 1995. Analysis of the PF numbers, taking into account corrections for $B_{0}$ and the varying seeing, indicates that the upcoming SC25 may have an amplitude of $118 \pm 29$, very similar to SC24. The results exclude amplitudes similar to or stronger than SC23, and make a peak similar to the Dalton cycles very unlikely. From the predicted SC25 amplitude, and based on flaring activity during the previous 4 solar cycles, a total of $745 \pm 58 \mathrm{M}$ - and X-class flares can be expected: $611 \pm 68$ M1_4 flares, $82 \pm 7$ M5_9 flares and $52 \pm 5 \mathrm{X}$-class events. Dropping the 0.7 rescaling factor which was used by NOAA/SWPC prior to GOES16, a total of 1151 M- and X-class flares can be expected: $957 \pm 72$ M1_4 flares, $118 \pm 17$ M5_9 flares and $76 \pm 1$ X-class events.

Acknowledgements. This work was supported by the SolarTerrestrial Centre of Excellence (STCE, http://www.stce.be/). The polar magnetic field data are from the Wilcox Solar Observatory (http://wso.stanford.edu/Polar.html) and are courtesy of J. T. Hoeksema. The Wilcox Solar Observatory is currently supported by NASA. International Sunspot Numbers were obtained from SILSO, World Data Center - Sunspot Number 
and Long-term Solar Observations, Royal Observatory of Belgium, http://www.sidc.be/SILSO/, 1976-2019. The "X-ray Flare" dataset was prepared by and made available through the NOAA National Geophysical Data Center (NGDC). White-light imagery courtesy of SOHO/MDI consortium. SOHO is a project of international cooperation between ESA and NASA. Figure 1 courtesy of NASA/SDO and the HMI science team. JHelioviewer is being developed as open source software by the ESA JHelioviewer Team. JHelioviewer is part of the ESA/NASA Helioviewer Project. The author would like to thank the two reviewers for their valuable comments that substantially improved this paper. A draft of this paper on the prediction for $\mathrm{SC} 25$ was submitted to the SC25 prediction panel in January 2019. The current paper predicts an amplitude for SC25 of $118 \pm 29$, very similar to the original $115 \pm 30$, despite significant corrections for $B_{0}$ and a slightly different formula for the seeing correction. The paper has also been updated with the additional polar faculae observations till March 2020, and expanded with the section on flare activity. The editor thanks two anonymous reviewers for their assistance in evaluating this paper.

\section{References}

Balogh A, Hudson HS, Petrovay K, von Steiger R. 2014. Introduction to the solar activity cycle: Overview of causes and consequences. Space Sci Rev 186(1-4): 1-15. https://doi.org/ $10.1007 / \mathrm{s} 11214-014-0125-8$.

Charbonneau P. 2020. Dynamo models of the solar cycle. Living Rev Sol Phys 17: 4. https://doi.org/10.1007/s41116-020-00025-6.

Chen AQ, Wang JX, Li JW, Feynman J, Zhang J. 2011. Statistical properties of superactive regions during solar cycles 19-23. A\&A 534: A47. https://doi.org/10.1051/0004-6361/201116790.

Clette F, Svalgaard L, Vaquero JM, Cliver EW. 2014. Revisiting the sunspot number. A 400-year perspective on the solar cycle. Space Sci Rev 186(1-4): 35-103. https://doi.org/10.1007/s11214-0140074-2.

Erofeev DV. 2001. Latitudinal distribution of polar faculae. Solar Phys 203(1): 9-25. https://doi.org/10.1023/A:1012741904366.

Georgieva K. 2011. Why the sunspot cycle is double peaked. Int. Sch. Res. Notices 2011: 437838. https://doi.org/10.5402/2011/ 437838.

Hathaway DH, Wilson RM, Reichmann EJ. 1994. The shape of the sunspot cycle. Solar Phys 151(1): 177-190. https://doi.org/ 10.1007/BF00654090.

Hirayama T, Moriyama F. 1979. Center to limb variation of the intensity of the photospheric faculae. Solar Phys 63: 251-255. https://doi.org/10.1007/BF00174531.

Hoeksema JT. 1995. The large-scale structure of the heliospheric current sheet during the ULYSSES epoch. Space Sci Rev 72(1-2): 137-148. https://doi.org/10.1007/BF00768770.

Janardhan P, Fujiki K, Ingale M, Bisoi SK, Rout D. 2018. Solar cycle 24: An unusual polar field reversal. $A \& A$ 618: A148. https://doi. org/10.1051/0004-6361/201832981.

Jenkins JL. 2009. The sun and how to observe it. Astronomers' Observing Guides. Springer Science+Business Media, LLC. ISBN 978-0-387-09497-7. https://doi.org/10.1007/978-0-387-09498-4.

Karak BB, Mandal S, Banerjee D. 2018. Double peaks of the solar cycle: an explanation from a dynamo model. Astrophys $J$ 866(1): 17. https://doi.org/10.3847/1538-4357/aada0d.
Kiepenheuer KO. 1964. Solar Site Testing. In: Le choix des sites d'observatoires astronomiques (site testing) [IAU symposium no. 19] tenu a Rome (Italie) du ler au 6 octobre 1962 avec le concours financier du Consiglio Nazionale delle richerche, Rosch J, (Ed.) International Astronomical Union. Symposium no. 19, Gauthier-Villars, Paris, 193 p. http://adsabs.harvard.edu/ abs/1964IAUS...19..193K.

Li K, Irie M, Wang J, Xiong S, Yun H, Liang H, Zhan L, Zhao H. 2002. Activity cycle of polar faculae. Publ Astron Soc Jpn 54(5): 787-792. https://doi.org/10.1093/pasj/54.5.787.

Meeus J. 1958. Une formule d'adoucissement pour l'activité solaire. Ciel et Terre 74: 445. http://adsabs.harvard.edu/abs/1958C\% 26T..74.445M.

Makarov VI, Makarova VV. 1996. Polar faculae and sunspot cycles. Solar Phys 163(2): 267-289. https://doi.org/10.1007/BF00148001.

Müller D, Nicula B, Felix S, Verstringe F, Bourgoignie B, Csillaghy A. 2017. JHelioviewer. Time-dependent 3D visualisation of solar and heliospheric data. A\&A 606: A10. https://doi.org/10.1051/ 0004-6361/201730893.

Muñoz-Jaramillo A, Sheeley NR, Zhang J, DeLuca EE. 2012. Calibrating 100 years of polar faculae measurements: Implications for the evolution of the heliospheric magnetic field. Astrophys $J$ 753(2): 146. https://doi.org/10.1088/0004-637X/753/2/146.

Muñoz-Jaramillo A, Dasi-Espuig M, Balmaceda LA, DeLuca EE. 2013. Solar cycle propagation, memory, and prediction: Insights from a century of magnetic proxies. Astrophys J Lett 767(2): L25. https://doi.org/10.1088/2041-8205/767/2/L25.

Norton AA, Gallagher JC. 2010. Solar-cycle characteristics examined in separate hemispheres: Phase, Gnevyshev gap, and length of minimum. Solar Phys 261: 193-207. https://doi.org/10.1007/ s11207-009-9479-6.

Pesnell WD. 2016. Predictions of solar cycle 24: How are we doing? Space Weather 14(1): 10-21. https://doi.org/10.1002/ 2015 SW001304.

Petrovay K. 2010. Solar cycle prediction. Living Rev Sol Phys 7(1): 6. https://doi.org/10.12942/lrsp-2010-6.

Petrovay K. 2020. Solar cycle prediction. Living Rev Sol Phys 17(1): 2. https://doi.org/10.1007/s41116-020-0022-z.

Poppe BB. 2000. New scales help public, technicians understand space weather. Eos Trans AGU 81(29): 322-328. https://doi.org/ 10.1029/00EO00247.

Saito K, Tanaka Y. 1957. Polar Faculae of the Sun. Publ Astron Soc Jpn 9: 106. http://adsabs.harvard.edu/abs/1957PASJ..9.106S.

Schatten KH, Scherrer PH, Svalgaard L, Wilcox JM. 1978. Using dynamo theory to predict the sunspot number during solar cycle 21. Geophys Res Lett 5: 411-414. https://doi.org/10.1029/ GL005i005p00411.

Schatten KH. 2005. Fair space weather for solar cycle 24. Geophys Res Lett 32(21): L21106. https://doi.org/10.1029/ 2005GL024363.

Scherrer PH, Bogart RS, Bush RI, Hoeksema JT, Kosovichev AG, Schou J, et al. 1995. The solar oscillations investigation Michelson doppler imager. Solar Phys 162(1-2): 129-188. https://doi.org/10.1007/BF00733429.

Schou J, Scherrer PH, Bush RI, Wachter R, Couvidat S, RabelloSoares MC, et al. 2012. Design and ground calibration of the helioseismic and magnetic imager (HMI) instrument on the solar dynamics observatory (SDO). Solar Phys 275(1-2): 229-259. https://doi.org/10.1007/s11207-011-9842-2.

Sheeley NR Jr. 1964. Polar faculae during the sunspot cycle. Astrophys J 140: 731. https://doi.org/10.1086/147966.

Sheeley NR Jr. 1966. Measurements of solar magnetic fields. Astrophys J 144: 723. https://doi.org/10.1086/148651. 
Sheeley NR Jr. 1976. Polar faculae during the interval 1906-1975. J Geophys Res 81(19): 3462. https://doi.org/10.1029/JA081i019p03462.

Sheeley NR Jr. 1991. Polar faculae - 1906-1990. Astrophys J, Part 1 (ISSN 0004-637X) 374: 386-389. https://doi.org/10.1086/ 170129.

Sheeley NR Jr. 2008. A century of polar faculae variations. Astrophys J 680(2): 1553-1559. https://doi.org/10.1086/588251.

Svalgaard L, Duvall TL Jr, Scherrer PH. 1978. The strength of the sun's polar fields. Solar Phys 58: 225-239. https://doi.org/ 10.1007/BF00157268.
Svalgaard L, Cliver EW, Kamide Y. 2005. Sunspot cycle 24: Smallest cycle in 100 years? Geophys Res Lett 32(1): L01104. https://doi.org/10.1029/2004GL021664.

Svanda M, Brun AS, Roudier T, Jouve L. 2016. Polar cap magnetic field reversals during solar grand minima: could pores play a role? A\&A 586: A123. https://doi.org/10.1051/0004-6361/201527314.

Tlatov AG. 2009. The minimum activity epoch as a precursor of the solar activity. Solar Phys 260(2): 465-477. https://doi.org/ 10.1007/s11207-009-9451-5.

Cite this article as: Janssens $\mathrm{J}$ 2021. Prediction of the amplitude of solar cycle 25 using polar faculae observations. J. Space Weather Space Clim. 11, 3. https://doi.org/10.1051/swsc/2020081. 